\title{
LYL1 gene amplification predicts poor survival of patients with uterine corpus endometrial carcinoma: analysis of the Cancer genome atlas data
}

Se $\mathrm{Kk} \mathrm{Kim}^{1 \dagger}{ }^{\dagger}$, Ji Won Lee ${ }^{2,3 \dagger}$, Nara Lee ${ }^{1}$, Maria Lee ${ }^{1 *}$, Hee Seung Kim ${ }^{1}$, Hyun Hoon Chung ${ }^{1}$, Jae-Weon Kim', Noh Hyun Park', Yong-Sang Song ${ }^{1}$ and Jeong-Sun $\mathrm{Seo}^{2,3,4^{*}}$

\begin{abstract}
Background: Somatic amplifications of the $L Y L 1$ gene are relatively common occurrences in patients who develop uterine corpus endometrial carcinoma (UCEC) as opposed to other cancers. This study was undertaken to determine whether such genetic alterations affect survival outcomes of UCEC.

Methods: In 370 patients with UCEC, we analysed clinicopathologic characteristics and corresponding genomic data from The Cancer Genome Atlas database. Patients were stratified according to LYL1 gene status, grouped as amplification or non-amplification. Heightened levels of cancer-related genes expressed in concert with LYL1 amplification were similarly investigated through differentially expressed gene and gene set enrichment analyses. Factors associated with survival outcomes were also identified.

Results: Somatic LYL1 gene amplification was observed in 22 patients (5.9\%) with UCEC. Patients displaying amplification (vs. non-amplification) were significantly older at the time of diagnosis and more often were marked by non-endometrioid, high-grade, or advanced disease. In survival analysis, the amplification subset showed poorer progression-free survival (PFS) and overall survival (OS) rates (3-year PFS: $34.4 \%$ vs. $79.9 \%, P=0.031 ; 5$-year OS: $25.1 \%$ vs. $84.9 \%, P=0.014)$. However, multivariate analyses adjusted for tumor histologic type, grade, and stage did not confirm LYL1 gene amplification as an independent prognostic factor for either PFS or OS. Nevertheless, MAPK, WNT, and cell cycle pathways were significantly enriched by LYL1 gene amplification $(P<0.001, P=0.002$, and $P=0.004$, respectively).

Conclusions: Despite not being identified as an independent prognostic factor in UCEC, LYL1 gene amplification is associated with other poor prognostic factors and correlated with upregulation of cancer-related pathways.
\end{abstract}

Keywords: Endometrial Neoplasms, The Cancer Genome Atlass, LYL1s, Survival analysiss, Gene expression pattern analysis, Gene set enrichment analysis

\section{Background}

Uterine corpus endometrial cancer (UCEC) imposes a global burden in both developed and developing countries [1]. In the United States, it is the most common gynecologic malignancy, accounting for 61,380

\footnotetext{
* Correspondence: marialeemd@gmail.com; jeongsun@snu.ac.kr

${ }^{\dagger}$ Equal contributors

${ }^{1}$ Department of Obstetrics and Gynecology, Seoul National University College of Medicine, Daehak-Ro, Jongno-Gu, Seoul, Republic of Korea ${ }^{2}$ Gongwu Genomic Medicine Institute (G2MI), Medical Research Center, Seoul National University Bundang Hospital, Seongnam, Republic of Korea Full list of author information is available at the end of the article
}

new cases in 2017 [2]. In Korea, the incidence of UCEC is clearly increasing and is estimated to comprise $2.5 \%$ (2578) of all new female cancers in 2017 [3, 4].

In 2013, The Cancer Genome Atlas (TCGA) Research Network issued an integrated report of genomic, transcriptomic, and proteomic profiles in 373 patients diagnosed with UCEC [5]. Furthermore, this consortium determined four prognostic categories (good $\rightarrow$ poor as shown) for classification of UCEC: (1) polymerase $\varepsilon$ (POLE) ultramutated; (2) microsatellite instability (MSI) hypermutated; (3) low copy number; and (4) high copy

(c) The Author(s). 2018 Open Access This article is distributed under the terms of the Creative Commons Attribution 4.0 International License (http://creativecommons.org/licenses/by/4.0/), which permits unrestricted use, distribution, and reproduction in any medium, provided you give appropriate credit to the original author(s) and the source, provide a link to the Creative Commons license, and indicate if changes were made. The Creative Commons Public Domain Dedication waiver (http://creativecommons.org/publicdomain/zero/1.0/) applies to the data made available in this article, unless otherwise stated. 
number. The high copy number group in particular includes most of the serous and serous-like endometrioid tumors, sharing genomic features with ovarian serous carcinomas. Researchers have since incorporated these molecular criteria into clinical trials designed to gauge postsurgical adjuvant treatment of UCEC (https://doi. org/10.1186/ISRCTN11659025).

In keeping with the era of precision medicine, discovery of reliable genetic changes is essential to provide individualized treatment of patients with $\operatorname{UCEC}[5,6]$. Little-known genes such as LYL1 may now be identified as novel prognostic indicators or as potential therapeutic targets. The LYL1 gene is located on the short (p) arm of chromosome 19 at position 13.13, where it encodes a protein implicated in blood vessel maturation and haematopoiesis [7]. As a member of basic helixloop-helix transcription factor family, the $L Y L 1$ gene is also known to regulate cell proliferation and differentiation [8], and a form of T-cell acute lymphoblastic leukaemia has been linked to a chromosomal aberration of LYL1 [7].

Curiously, somatic amplifications of the LYL1 gene frequently accompany UCEC, more so than most other cancers, ranking second among TCGA listings. However, its ramifications in this setting have yet to be fully explored. The current study, entailing TCGA database analysis, was undertaken to determine whether genetic alterations in the $L Y L 1$ gene (such as amplification) may impact survival outcomes in patients with UCEC.

\section{Methods}

\section{Data acquisition}

We downloaded genomic alteration data on patients with UCEC and corresponding clinicopathologic profiles at the Genomics Data Commons (https://portal. gdc.cancer.gov) and cBioPortal for Cancer Genomics (http://www.cbioportal.org) web portals. The Illumina Genome Analyzer served as platform for DNA sequencing (Illumina Inc., San Diego, CA, USA). This study complied with TCGA publication guidelines and policies (http:// cancergenome.nih.gov/publications/publicationguidelines). The Institutional Review Board of Seoul National University Hospital ruled that no formal ethics approval was required in this study.

\section{Study population}

In total, 370 patients with UCEC qualified for this study. The clinicopathologic data collected included age, underlying comorbidities, International Federation of Gynecology and Obstetrics (FIGO) stage, tumor histologic type and grade, and treatment of UCEC (ie, surgery, radiation, chemotherapy). Tumor MSI status was also collected. Patients were assigned to $L Y L 1$ gene amplification and non-amplification groups as warranted.

\section{Bioinformatics analysis}

LYL1 gene status, especially whether it was amplified, was determined through the cBioPortal for Cancer Genomics (http://www.cbioportal.org). Level-3 data of patients with UCEC and raw reads (HTSeq-counts) of differentially expressed gene (DEG) analyses were accessed via FireBrowse (http://firebrowse.org). The Kyoto Encyclopedia of Genes and Genomes (KEGG) pathway analysis of gene expression data [9] was subjected to Gene Set Enrichment Analysis (GSEA) [10]. For visualization of enrichment pathway, the NetworkAnalyst (http://www.networkanalyst.ca) was used [11].

In doing so, the Search Tool for the Retrieval of Interacting Genes/Proteins (STRING) database was applied, achieving confidence scores of 400-1000 [12]. DEGs were identified through open-source software analysis (R package DESeq2; http://www.bioconductor.org) [13].

\section{Statistical analysis}

To compare clinicopathologic features of the two patient subsets, Student's $t$-test and Mann-Whitney $U$-test were applied to continuous variables, and Pearson's chisquared and Fisher's exact tests for categorical variables.

We defined PFS as the time elapsed between date of initial diagnosis and date of disease progression, whereas overall survival (OS) represented the time interval between date of initial diagnosis and date of cancer-related death or end of study. Survival estimates were generated via Kaplan-Meier method and log-rank test. Cox proportional hazards regression models were engaged to calculate hazard ratios (HRs) and 95\% confidence intervals (CIs). For survival analytics, we relied on commercially available software (SPSS v21.0; IBM, Armonk, NY, USA). Opensource programming ( $\mathrm{R}$ v2.12.1, ISBN 3-900,051-07-0, http://www.R-project.org; R Foundation for Statistical Computing, Vienna, Austria) was used for all other computations. Statistical significance was set at $P<0.05$.

\section{Results}

\section{Somatic copy number variations in UCEC}

Frequencies of somatic amplifications involving the LYL1 gene are depicted according to TCGA classification in Fig. 1a. UCEC ranked second among cancers in terms of LYL1 gene amplification. In genomic alteration analyses, chromosomes 1q, 3q, 8q, 17q, and $19 p$ were frequently amplified in this patient population (Fig. 1b). The LYL1 gene of $19 \mathrm{p}$ arm was amplified in 5.9\% (22/370) of patients with UCEC. Additionally, the LYL1 gene was one of the 15 mostly amplified oncogenes filtered by gene family in GSEA (Fig. 1c). Meanwhile, the 15 mostly deleted tumor suppressor genes, including PTEN, are displayed in Fig. 1d. 


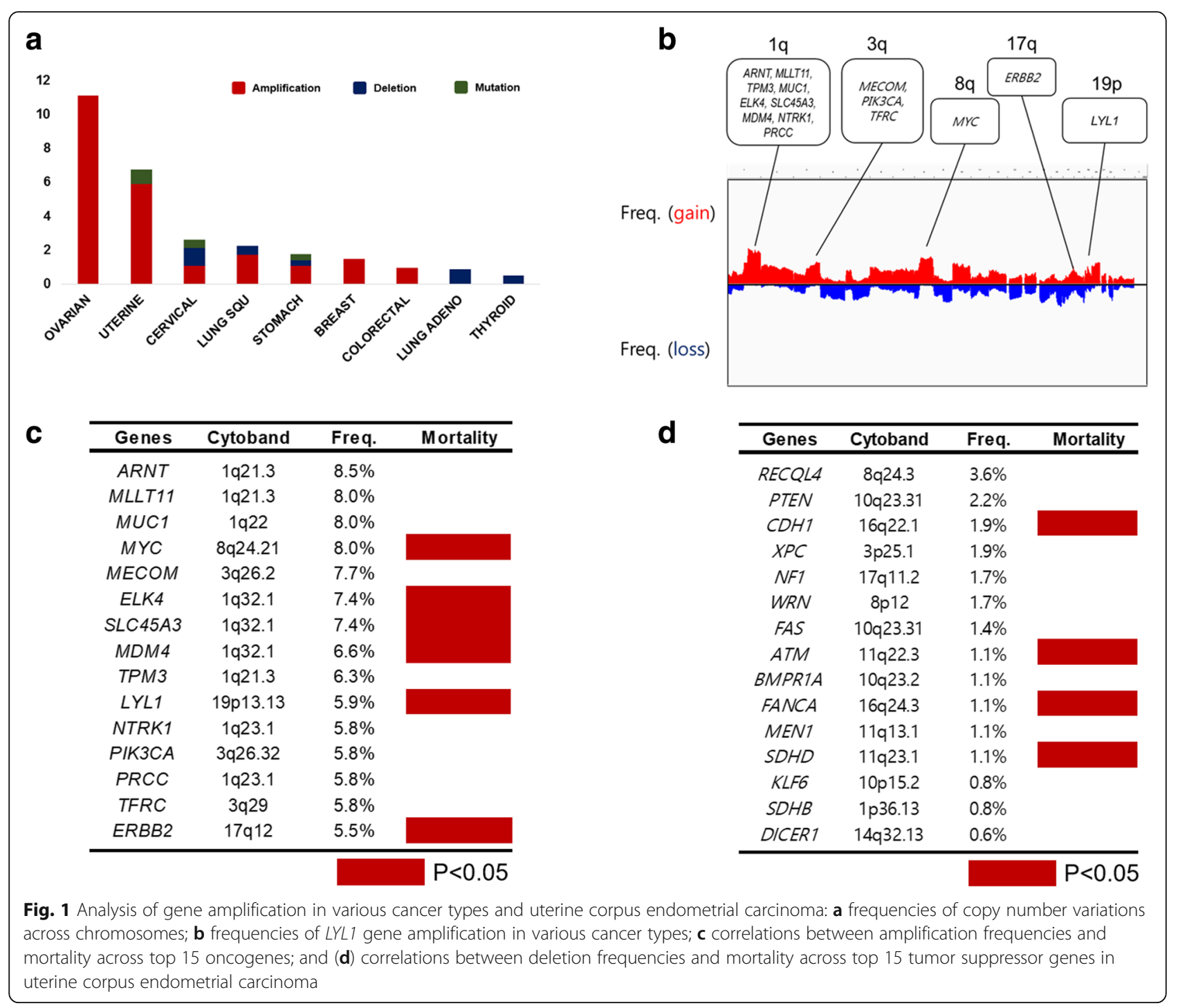

\section{Characteristics of patients with UCEC}

Patients' clinicopathologic characteristics are presented in Table 1. Mean patient age was 63 years. Of the 370 patient participants, 304 (82.2\%), 52 (14.1\%), and 14 (3.8\%) displayed endometrioid, serous, and mixed histologic types of UCEC, respectively. Members of the LYL1 amplification (vs. non-amplification) group were significantly older at time of diagnosis and more often exhibited biologically aggressive tumors, marked by advanced-stage disease (FIGO stage III-IV; $P=0.003$ ), high-grade malignancy (grade 3; $P<0.001)$, and serous histologic type $(P<0.001)$. Proportions of the four TCGA categories of UCEC also showed comparative differences, with $72.7 \%$ of amplification group members achieving high copy number rank, versus $12.1 \%$ in the non-amplification group $(P<0.001)$. In terms of adjuvant treatment, chemotherapy recipients were more numerous in $L Y L 1$ amplification group than in non-amplification group $(50.0 \%$ vs $28.4 \% ; P=0.032)$ (Table 1).

\section{Between-group comparisons of survival outcomes and identification of prognostic factors}

During the observation period (median, 23.9 months; range, 0.5-191.7 months), 5 patients in the amplification group and 34 in the non-amplification group died of their disease. Survival analysis indicated poorer 3-year PFS $(34.4 \%$ vs. $79.9 \% ; P=0.031)$ and 5 -year OS $(25.1 \%$ vs. $84.9 \% ; P=0.014$ ) in the amplification (vs. nonamplification) group (Fig. 2).

LYL1 gene amplification also showed a significant association with poor OS in univariate analysis $(P=0.019)$ (Table 2). However, after adjusting for variables such as histologic type, grade, and FIGO stage, LYL1 gene status was not confirmed as a significant prognostic factor in 
Table 1 Clinicopathologic characteristics of patients

\begin{tabular}{|c|c|c|c|c|}
\hline Characteristics & All $(n=370, \%)$ & LYL1 amplification $(n=22, \%)$ & LYL1 non-amplification $(n=348, \%)$ & $P$ \\
\hline Age, years & & & & $<0.001$ \\
\hline Mean \pm SD & $63.1 \pm 11.0$ & $72.7 \pm 8.0$ & $62.5 \pm 10.9$ & \\
\hline Menopause $^{a}$ & & & & 0.393 \\
\hline Yes & $313(84.6)$ & $0(0)$ & $27(7.8)$ & \\
\hline No & $27(7.3)$ & $21(95.5)$ & $292(83.9)$ & \\
\hline Unknown & $30(8.1)$ & $1(4.5)$ & $29(8.3)$ & \\
\hline Diabetes & & & & 0.774 \\
\hline Yes & $83(22.4)$ & $5(22.7)$ & $78(22.4)$ & \\
\hline No & $221(59.7)$ & $11(50.0)$ & $210(60.3)$ & \\
\hline Unknown & $66(17.8)$ & $6(27.3)$ & $60(17.2)$ & \\
\hline Hypertension & & & & 0.420 \\
\hline Yes & $195(52.7)$ & $11(50.0)$ & $184(52.9)$ & \\
\hline No & $135(36.5)$ & $5(22.7)$ & $130(37.4)$ & \\
\hline Unknown & $40(10.8)$ & $6(27.3)$ & $34(9.8)$ & \\
\hline Histologic type & & & & $<0.001$ \\
\hline Endometrioid & $304(82.2)$ & $6(27.3)$ & $298(85.6)$ & \\
\hline Serous & $52(14.1)$ & $13(59.1)$ & $39(11.2)$ & \\
\hline Mixed & $14(3.8)$ & $3(13.6)$ & $11(3.2)$ & \\
\hline Grade & & & & $<0.001$ \\
\hline 1 & $88(23.8)$ & $0(0)$ & $88(25.3)$ & \\
\hline 2 & $106(28.6)$ & $1(4.5)$ & $105(30.2)$ & \\
\hline 3 & $176(47.6)$ & $21(95.5)$ & $155(44.5)$ & \\
\hline FIGO stage & & & & 0.003 \\
\hline । & $254(68.6)$ & $9(40.9)$ & $245(70.4)$ & \\
\hline$\|$ & $24(6.5)$ & $2(9.1)$ & $22(6.3)$ & \\
\hline III & $72(19.5)$ & $9(40.9)$ & $63(18.1)$ & \\
\hline IV & $17(4.6)$ & $2(9.1)$ & $15(4.3)$ & \\
\hline Unknown & $3(0.8)$ & $0(0)$ & $3(0.9)$ & \\
\hline \multicolumn{5}{|l|}{ TCGA type } \\
\hline POLE ultra-mutated & $17(4.6)$ & $0(0)$ & $17(4.9)$ & 0.612 \\
\hline MSI hyper-mutated & 65 (17.6) & $0(0)$ & $65(18.7)$ & 0.019 \\
\hline Low copy number & $90(24.3)$ & $0(0)$ & $90(25.9)$ & 0.006 \\
\hline High copy-number & $58(15.7)$ & $16(72.7)$ & $42(12.1)$ & $<0.001$ \\
\hline Indeterminate & $140(37.8)$ & $6(27.3)$ & $134(38.5)$ & 0.292 \\
\hline MSI status & & & & $<0.001$ \\
\hline Stable & $223(60.2)$ & $22(100.0)$ & $201(57.8)$ & \\
\hline Low & $19(5.1)$ & $0(0)$ & $19(5.5)$ & \\
\hline High & $125(33.7)$ & $0(0)$ & $125(35.9)$ & \\
\hline Indeterminate & $3(0.8)$ & $0(0)$ & $3(0.9)$ & \\
\hline \multicolumn{5}{|l|}{ Adjuvant treatment } \\
\hline Chemotherapy only & $110(29.7)$ & $11(50.0)$ & $99(28.4)$ & 0.032 \\
\hline Radiation only & $55(14.9)$ & $2(9.1)$ & $53(15.2)$ & 0.756 \\
\hline Chemotherapy + Radiation & $23(6.2)$ & $1(4.5)$ & $22(6.3)$ & 1.000 \\
\hline Hormone therapy & $17(4.6)$ & $0(0)$ & $17(4.9)$ & 0.612 \\
\hline
\end{tabular}

Abbreviations: FIGO International Federation of Gynecology and Obstetrics, TCGA The Cancer Genome Atlas; POLE polymerase $\varepsilon$, MSI microsatellite instability, SD standard deviation

${ }^{\mathrm{a}}$ Menopause was defined as amenorrhea for 6 months or more 

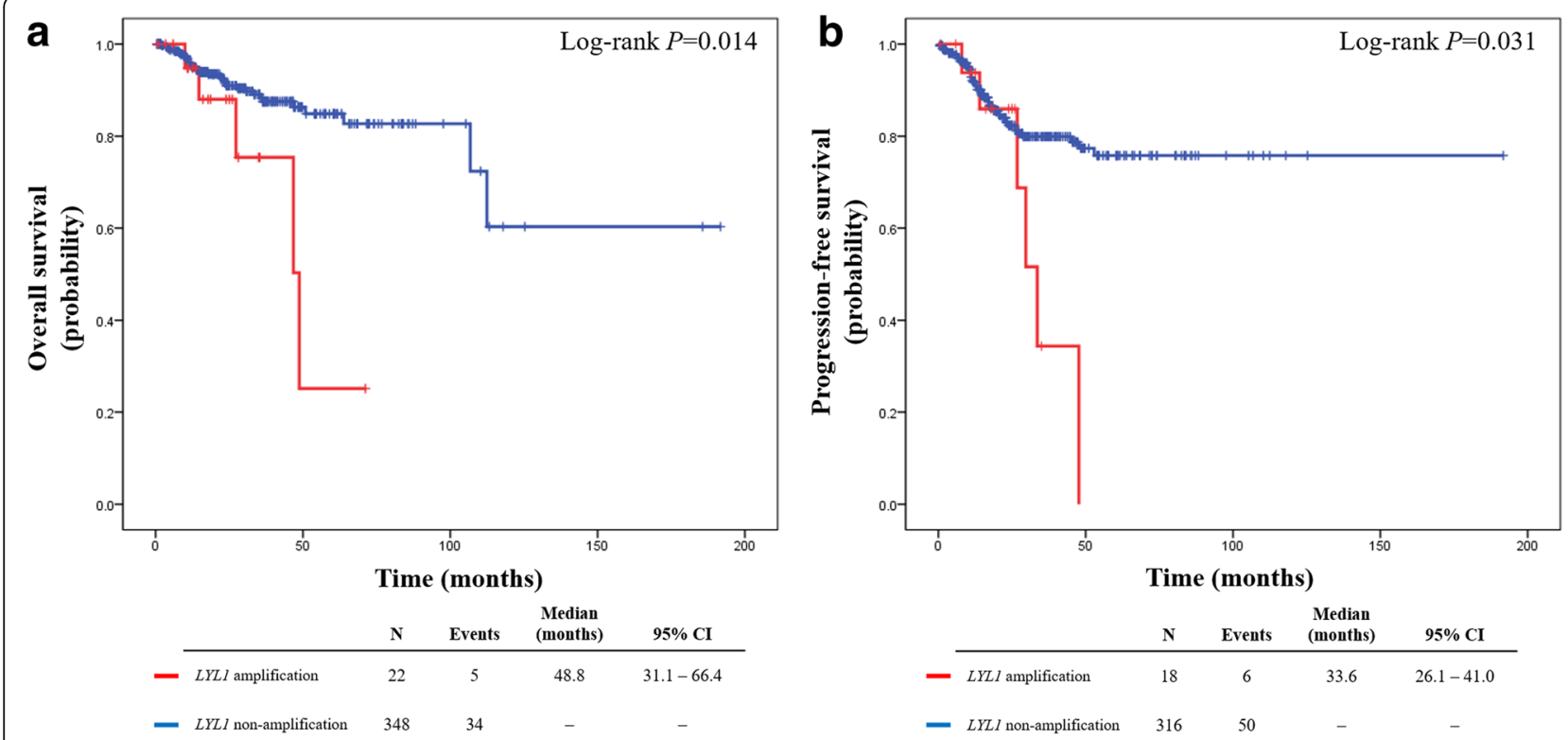

Fig. 2 Survival outcomes of patients with uterine corpus endometrial carcinoma, shown by LYL1 gene status: a overall survival and (b) progression-free survival

OS. Only advanced-stage disease (FIGO stage III-IV) emerged as an independent predictor of poor prognosis (adjusted HR, 3.509; 95\% CI, 1.734-7.101; $P<0.001$ ). Table 2 also presents factors associated with PFS. In univariate analysis, $L Y L 1$ gene amplification was associated with poor PFS $(P=0.037)$, but its statistical significance was not sustained in multivariate analysis. Advancedstage disease (FIGO stage III-IV) was identified as an independent poor prognostic factor for PFS (adjusted HR, 3.581; 95\% CI, 1.981-6.473; $P<0.001$ ).

We also stratified patients by tumor histologic type for subgroup analysis. In those with endometrioid cancers $(n=304)$, neither PFS $(P=0.070)$ nor OS $(P=0.323)$ differed significantly by $L Y L 1$ gene status (amplification vs. non-amplification). However, results of multivariate analysis showed a trend towards worse PFS in the patients with LYL1 gene amplification (adjusted HR, 4.093; 95\% CI, 0.926-18.012; $P=0.063$ ) (Table 3).

\section{DEGs in LYL1 amplified tumors}

We performed GSEA pathway analysis of 993 genes showing increased levels of expression in conjunction with LYL1 amplification. Consequently, we found significant upregulation of MAPK $(P<0.001)$, WNT $(P=0.002)$, cell cycle $(P=0.004)$, and cancer-related $(P<0.001)$ pathways (Fig. 3a, b). Of 993 DEGs, 384 cancer-related genes filtered via STRING database were enriched through these pathways. MYC, CDK6, $P R K A C A$, and $E R B B 2$ genes were found to frequently interact with other cancer-related genes (Fig. 3c).
We also conducted GSEA according to histologic types and TCGA classes (Additional file 1: Figure S1). Among the four TCGA classes, only the high copy number group showed $L Y L 1$ amplifications, and cell proliferation pathway was significantly enriched in this group. Compared to endometrioid type, cancer-related and cell proliferation pathways and genes were more commonly enriched in serous type (Additional file 2: Figure S2).

\section{Discussion}

In the present study, we used TCGA database analysis to determine the potential impact of $L Y L 1$ gene amplification on survival outcomes in patients with UCEC. Although patients displaying LYL1 gene amplification showed poorer PFS and OS compared to those with non-amplification, multi-variate analyses failed to prove it as an independent prognostic factor.

A number of studies have been similarly conducted to date to identify novel biomarkers for patient survival in various types of cancer. In particular, the prognostic impact made by altered expression levels of L1CAM and $M Y C$, both homeobox gene family members, has been researched through TCGA database analysis [14-16]. The $L Y L 1$ gene, a basic helix-loop-helix transcription factor and a known oncogene in human and mouse cancers, is linked to many cancer-related properties, such as angiogenesis [17-19]. Through genetic and epigenetic modulations, the $L Y L 1$ gene acts to regulate cell proliferation and differentiation [8]. Both in vivo and in vitro experiments have also demonstrated its interactions with 


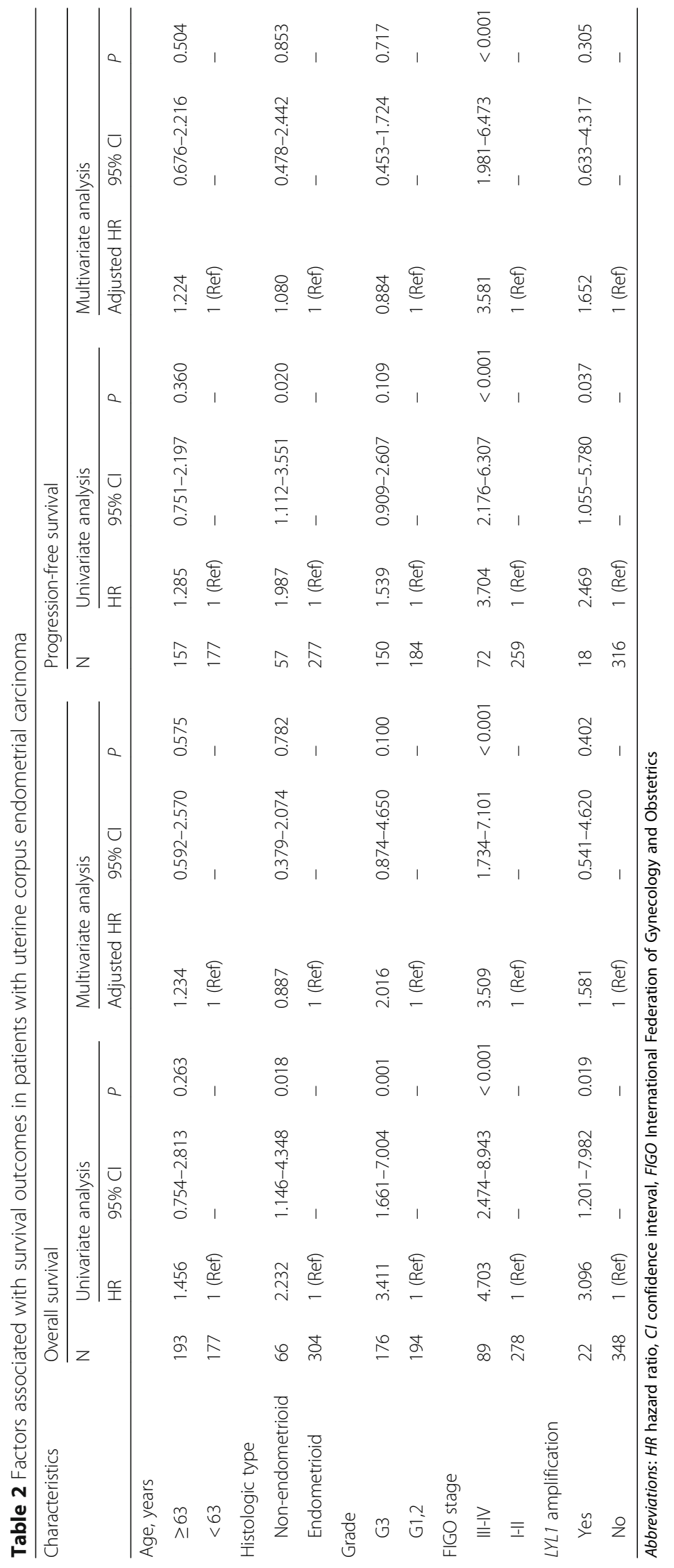


Kim et al. BMC Cancer (2018) 18:494

Page 7 of 10

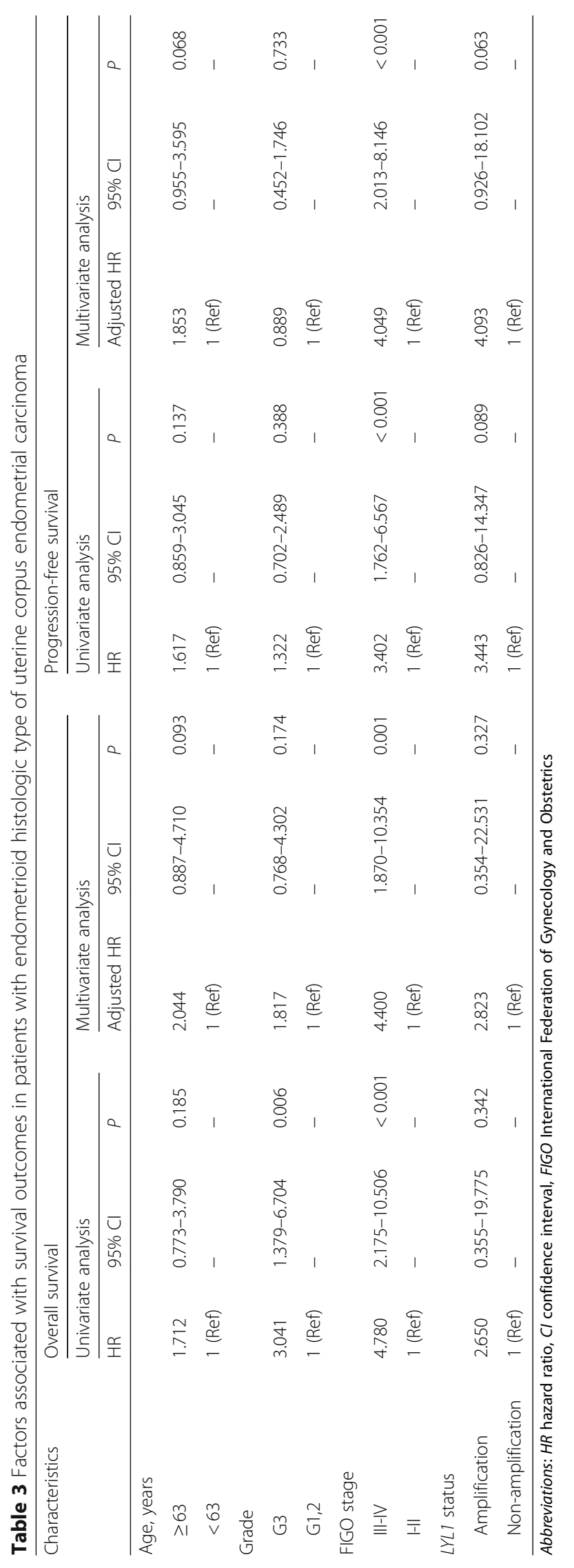




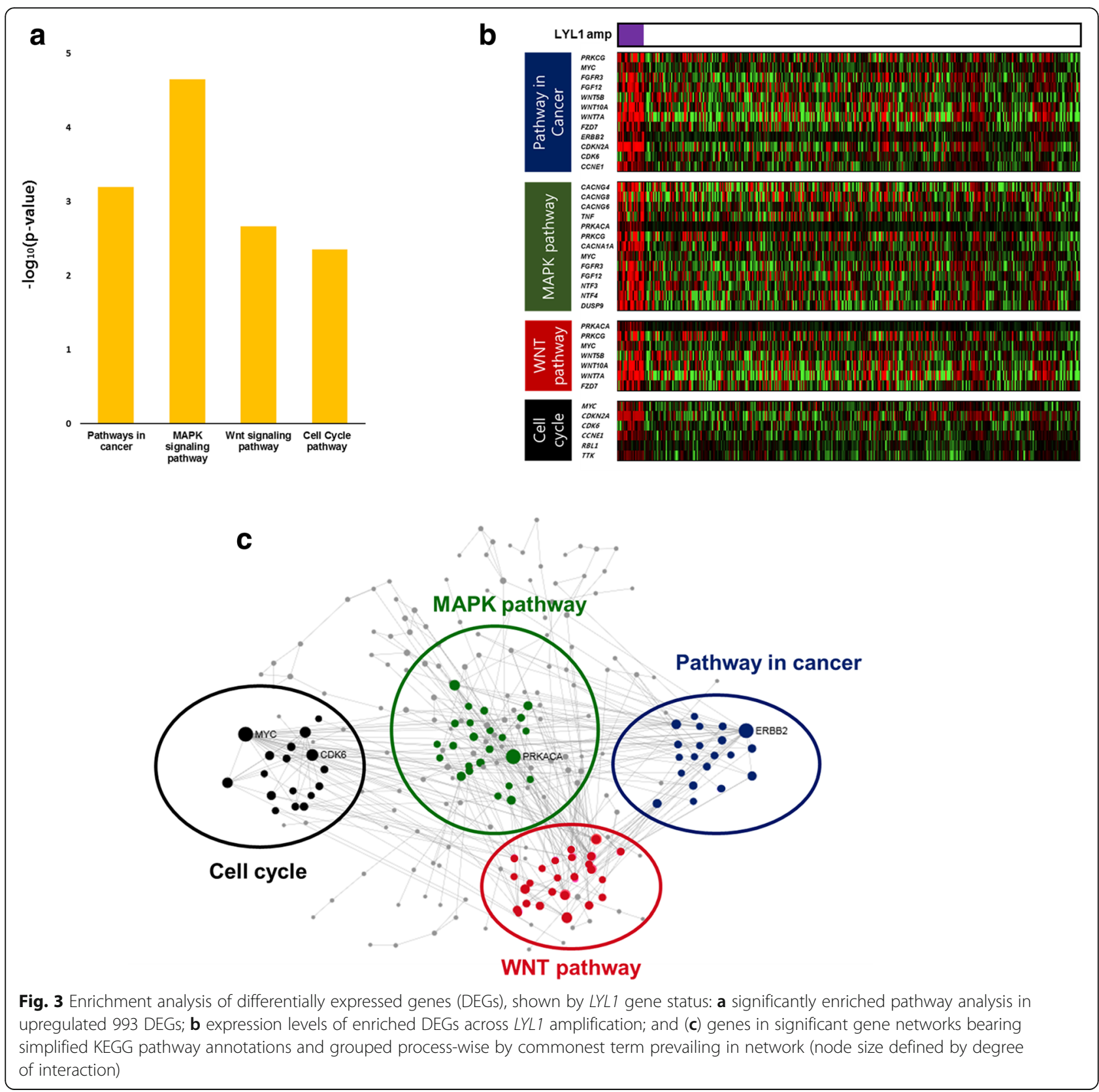

various oncogenes, such as MYC, TAL1, TAL2, and LMO2 [20, 21].

Through our TCGA data analysis of LYL1 gene amplification in patients with UCEC, we discovered that overexpressed cancer-related genes are enriched by MAPK, WNT, and cell cycle pathways in such patients. Specifically, $M Y C, C D K 6, P R K A C A$, and ERBB2, all well-known oncogenes and cancer markers, were overexpressed in conjunction with $L Y L 1$ gene amplification. Both $M Y C$ and $E R B B 2$ have likewise shown associations with uterine cancers in earlier studies [22-26]. Additionally, expression of PRKACA was positively correlated with $L Y L 1$ amplification (Pearson's coefficient (r), 0.442).
Unfortunately, only advanced-stage disease emerged as a significant marker of poor prognosis in multivariate analyses. $L Y L 1$ gene amplification was not identified as an independent prognostic factor. However, most of our cohort had early-stage disease (FIGO stages I and II: $68.6 \%$ and $6.5 \%$, respectively). According to Surveillance, Epidemiology, and End Results data of the National Cancer Institute, the 5-year survival rate for UCEC with distant metastasis is a dismal $16.2 \%$, compared with $95.3 \%$ for disease confined to primary sites [27]. It is thus apparent that the stage of UCEC impacts survival outcomes dramatically, hindering analysis of amplification effects in the current study population. 
The current study has several acknowledged limitations, the first being that associations between the LYL1 gene and other genes or genetic mechanisms were not validated, and the proteins expressed were not measured. Such proteogenomic studies would perhaps underscore the effects of these genetic alterations and the accuracy and completeness of genomic profiling. In addition, further efforts to identify the genetic and epigenetic regulatory mechanisms of the LYL1 gene and an evaluation of its efficacy as a prognostic indicator and therapeutic target are warranted. In UCEC cell lines, the LYL1 gene could be overexpressed or inhibited by siRNA, determining subsequent flux in cell differentiation, proliferation, or death. A LYL1 gene knock-out patient-derived xenograft animal model is one possible investigative approach. Another limitation was the sample size of the LYL1 gene amplification group $(n=22)$, which was too small for reasonable statistical inferences. Despite these drawbacks, we were able to explore the prognostic potential of the novel LYL1 gene in the setting of UCEC using both TCGA and clinicopathologic data. LYL1 gene amplification and its association with expression levels of other genes were demonstrated as well.

\section{Conclusions}

In conclusion, LYL1 gene amplification is not identified as an independent prognostic factor in UCEC. However, we discovered that cancer-related pathways, such as MAPK, WNT, and cell cycle pathways are upregulated in patients with $L Y L 1$ amplification. Correlations between LYL1 amplification and increased expression levels of cancer-related genes (MYC, CDK6, PRKACA, and $E R B B 2)$ are also observed. Its potential for prognostic indicator and therapeutic targeting may be implied based on overexpression of such affiliated oncogenes. Additional multi-omics and genome-wide data studies are warranted.

\section{Additional files}

Additional file 1: Figure S1. Gene set enrichment analysis according to histologic types and TCGA classes. (PNG $144 \mathrm{~kb}$ )

Additional file 2: Figure S2. Enriched genes of cancer-related and cell proliferation pathways according to the two histologic types; serous and endometrioid. (PNG 486 kb)

\section{Abbreviations}

Cl: Confidence interval; DEG: Differentially expressed gene; HR: Hazard ratio; KEGG: The kyoto encyclopedia of genes and genomesGSEAGene set enrichment analysis; MSI: Microsatellite instabilityFIGOInternational Federation of Gynecology and Obstetrics; OS: Overall survival; PFS: Progression-free survival; POLE: Polymerase $\varepsilon$; STRING: The search tool for the retrieval of interacting genes/proteins; TCGA: The cancer genome atlas; UCEC: Uterine corpus endometrial cancer

\section{Availability of data and materials}

The datasets used and/or analysed during the current study were downloaded at the Genomics Data Commons (https://portal.gdc.cancer.gov) and cBioPortal for Cancer Genomics (http://www.cbioportal.org) web portals.

\section{Authors' contributions}

ML, YSS, and JSS contributed to the study conception and design. SIK, JWL, and $\mathrm{NL}$ analysed and interpreted the data. SIK, JWL, and ML were major contributors in writing the manuscript. HSK, HHC, JWK, and NHP involved in drafting the manuscript and revising it. All authors have read and approved the original and revised versions of the manuscript, as well as the final manuscript.

\section{Ethics approval and consent to participate}

This study complied with TCGA publication guidelines and policies (http://cancergenome.nih.gov/publications/publicationguidelines). A local ethics committee, the Seoul National University Hospital Institutional Review Board, ruled that no formal ethics approval was required in this particular case.

\section{Competing interests}

The authors declare that they have no competing interests.

\section{Publisher's Note}

Springer Nature remains neutral with regard to jurisdictional claims in published maps and institutional affiliations.

\section{Author details}

${ }^{1}$ Department of Obstetrics and Gynecology, Seoul National University College of Medicine, Daehak-Ro, Jongno-Gu, Seoul, Republic of Korea. ${ }^{2}$ Gongwu Genomic Medicine Institute (G2MI), Medical Research Center, Seoul National University Bundang Hospital, Seongnam, Republic of Korea. ${ }^{3}$ Department of Biomedical Sciences, Seoul National University College of Medicine, Seoul, Republic of Korea. ${ }^{4}$ Macrogen Inc., Seoul, Republic of Korea.

Received: 13 February 2018 Accepted: 23 April 2018

Published online: 02 May 2018

References

1. Fitzmaurice C, Dicker D, Pain A, Hamavid H, Moradi-Lakeh M, Maclntyre MF, et al. The global burden of Cancer 2013. JAMA Oncol. 2015;1:505-27.

2. Siegel RL, Miller KD, Jemal A. Cancer statistics, 2017. CA Cancer J Clin. 2017;67:7-30.

3. Lim MC, Moon EK, Shin A, Jung KW, Won YJ, Seo SS, et al. Incidence of cervical, endometrial, and ovarian cancer in Korea, 1999-2010. J Gynecol Oncol. 2013;24:298-302.

4. Jung KW, Won YJ, Oh CM, Kong HJ, Lee DH, Lee KH. Prediction of Cancer incidence and mortality in Korea, 2017. Cancer Res Treat. 2017:49:306-12.

5. Cancer Genome Atlas Research N, Kandoth C, Schultz N, Cherniack AD, Akbani R, Liu Y, et al. Integrated genomic characterization of endometrial carcinoma. Nature. 2013;497:67-73.

6. Hodson R. Precision medicine. Nature. 2016;537:S49.

7. LYL1 [Internet]. Bethesda (MD): National Library of Medicine (US), National Center for Biotechnology Information; 2004 - [cited 2018 Jan 30]. Available from: https://www.ncbi.nlm.nih.gov/gene/4066/.

8. San-Marina S, Han Y, Suarez Saiz F, Trus MR, Minden MD. Lyl1 interacts with CREB1 and alters expression of CREB1 target genes. Biochim Biophys Acta. 2008;1783:503-17.

9. Kanehisa M, Furumichi M, Tanabe M, Sato Y, Morishima K. KEGG: new perspectives on genomes, pathways, diseases and drugs. Nucleic Acids Res. 2017;45:D353-61.

10. Subramanian A, Tamayo P, Mootha VK, Mukherjee S, Ebert BL, Gillette MA, et al. Gene set enrichment analysis: a knowledge-based approach for interpreting genome-wide expression profiles. Proc Natl Acad Sci U S A. 2005;102:15545-50.

11. Xia J, Gill EE, Hancock RE. NetworkAnalyst for statistical, visual and network-based meta-analysis of gene expression data. Nat Protoc. 2015;10:823-44.

12. Szklarczyk D, Franceschini A, Wyder S, Forslund K, Heller D, Huerta-Cepas J, et al. STRING v10: protein-protein interaction networks, integrated over the tree of life. Nucleic Acids Res. 2015;43:D447-52.

13. Varet H, Brillet-Gueguen L, Coppee JY, Dillies MA. SARTools: a DESeq2- and EdgeR-based R pipeline for comprehensive differential analysis of RNA-Seq data. PLoS One. 2016;11:e0157022. 
14. Dellinger TH, Smith DD, Ouyang C, Warden CD, Williams JC, Han ES. LICAM is an independent predictor of poor survival in endometrial cancer - an analysis of the Cancer genome atlas (TCGA). Gynecol Oncol. 2016;141:336-40.

15. Eoh KJ, Kim HJ, Lee JY, Nam EJ, Kim S, Kim SW, et al. Upregulation of homeobox gene is correlated with poor survival outcomes in cervical cancer. Oncotarget. 2017;8:84396-402.

16. Cancer Genome Atlas Research N. Integrated genomic analyses of ovarian carcinoma. Nature. 2011;474:609-15.

17. Meng YS, Khoury H, Dick JE, Minden MD. Oncogenic potential of the transcription factor LYL1 in acute myeloblastic leukemia. Leukemia. 2005:19:1941-7.

18. Pirot N, Deleuze V, El-Hajj R, Dohet C, Sablitzky F, Couttet P, et al. LYL1 activity is required for the maturation of newly formed blood vessels in adulthood. Blood. 2010;115:5270-9.

19. Orsulic S, Li Y, Soslow RA, Vitale-Cross LA, Gutkind JS, Varmus HE. Induction of ovarian cancer by defined multiple genetic changes in a mouse model system. Cancer Cell. 2002;1:53-62.

20. Bain G, Engel I, Robanus Maandag EC, te Riele HP, Voland JR, Sharp LL, et al. E2A deficiency leads to abnormalities in alphabeta T-cell development and to rapid development of T-cell lymphomas. Mol Cell Biol. 1997;17:4782-91.

21. Deleuze V, El-Hajj R, Chalhoub E, Dohet C, Pinet V, Couttet P, et al. Angiopoietin-2 is a direct transcriptional target of TAL1, LYL1 and LMO2 in endothelial cells. PLoS One. 2012;7:e40484.

22. Li L, Osdal T, Ho Y, Chun S, McDonald T, Agarwal P, et al. SIRT1 activation by a c-MYC oncogenic network promotes the maintenance and drug resistance of human FLT3-ITD acute myeloid leukemia stem cells. Cell Stem Cell. 2014;15:431-46.

23. Tadesse S, Yu M, Kumarasiri M, Le BT, Wang S. Targeting CDK6 in cancer: state of the art and new insights. Cell Cycle. 2015;14(20):3220-30.

24. Martinez-Ledesma E, Verhaak RG, Trevino V. Identification of a multi-cancer gene expression biomarker for cancer clinical outcomes using a network-based algorithm. Sci Rep. 2015;5:11966.

25. Elsahwi KS, Santin AD. erbB2 overexpression in uterine serous Cancer: a molecular target for Trastuzumab therapy. Obstet Gynecol Int. 2011; https://doi.org/10.1155/2011/128295.

26. Subramaniam KS, Omar IS, Kwong SC, Mohamed Z, Woo YL, Mat Adenan NA et al. Cancer-associated fibroblasts promote endometrial cancer growth via activation of interleukin-6/STAT-3/C-Myc pathway. Am J Cancer Res. 2016;6:200-13

27. National Cancer Institute. SEER Research Data 1973-2014 [Internet]. Surveillance Research Program, Surveillance Systems Branch; 2017 [cited 2018 Jan 30]. Available from: https://www.seer.cancer.gov/.

\section{Ready to submit your research? Choose BMC and benefit from:}

- fast, convenient online submission

- thorough peer review by experienced researchers in your field

- rapid publication on acceptance

- support for research data, including large and complex data types

- gold Open Access which fosters wider collaboration and increased citations - maximum visibility for your research: over $100 \mathrm{M}$ website views per year

At BMC, research is always in progress.

Learn more biomedcentral.com/submissions 